\title{
Estudio exploratorio de la calidad de vida en el trabajo de ordeñadores de sistemas de producción de leche
}

\author{
Vásquez-Jaramillo, C. @; Barrios, D. y Cerón-Muñoz, M.F.
}

Facultad de Ciencias Agrarias. Universidad de Antioquia. Medellín. Colombia.

Palabras CLAVE ADICIONALES

Agronegocios.

Competitividad.

Conducta social.

Gestión humana.

\section{RESUMEN}

La política comercial se ha orientado hacia una mayor apertura e integración de mercados globales, lo que ha creado en el sector lechero la necesidad de ser cada vez más competitivo. La gestión de talento humano constituye el factor clave para la creación de ventajas y el desarrollo de la estrategia empresarial. El objetivo fue explorar la calidad de vida en el trabajo y los factores asociados a la calidad de vida en el trabajo de ordeñadores en lecherías especializadas. Se encuestaron 66 colaboradores de labores de ordeño en sistemas de producción de leche de Antioquia, Colombia. Para el análisis estadístico se construyeron dos ecuaciones estructurales mediante análisis de factor exploratorio y confirmatorio; adicionalmente se analizó la variable "deseo de permanecer" mediante un análisis de partición recursiva y árboles de regresión. Los resultados mostraron en general un buen nivel de calidad de vida laboral. Además, se encontró que la variable percepción de riesgo es la que influye principalmente en la intención de cambiar de trabajo, mientras que la similitud salarial frente a otras fincas de la zona marca la intención de permanencia en el trabajo actual. Este trabajo esclarece un punto de partida en el diagnóstico de elementos de gestión humana, que permita mejorar la toma de decisiones, el desempeño productivo y la competitividad de los sistemas agropecuarios.

\section{Exploratory study of the quality of work life of milkers of dairy production systems}

\section{SUMMARY}

\section{ADDITIONAL KEYWORDS}

\section{Agribusiness.}

Competitiveness.

Human management.

Social behavior.

\section{INFORMATION}

\section{Cronología del artículo.}

Recibido/Received: 17.01.2017

Aceptado/Accepted: 06.03.2018

On-line: 15.04 .2018

Correspondencia a los autores/Contact e-mail:

grupogamma@udea.edu.co

\section{INTRODUCCIÓN}

El trabajo es una faceta importante en la vida de las personas porque una gran parte del tiempo lo dedican a actividades laborales y genera una serie de compensaciones económicas, materiales, psicológicas y sociales que le permiten lograr su propia identidad y su rol en la sociedad, contribuyendo a la creación de bienes y servicios (González et al., 2002). Partiendo de esto, y de la ne-
The commercial policy has been oriented toward greater openness and integration of global markets, which has created in the dairy sector the need to be more and more competitive. The management of human talent is the key factor for the creation of advantages and the development of business strategy. The objective was to explore the quality of life at work and the factors associated to the quality of life in the work of milkers in specialized dairies. We surveyed 66 coworkers in milk production systems in Antioquia, Colombia. For the statistical analysis, two structural equations were constructed by exploratory and confirmatory factor analysis; Additionally, the variable "desire to remain" was analyzed through a recursive partition analysis and regression trees. The results showed in general a good level of quality of working life. In addition, it was found that the variable risk perception is the one that influences mainly the intention to change work, while the salary similarity to other farms in the area marks the intention of permanence in the current job. This work clarifies a starting point in the diagnosis of elements of human management, that allows to improve the decision making, the productive performance and the competitiveness of the agricultural systems cesidad de administrar de manera efectiva los recursos humanos dentro de las organizaciones, nace la gestión del talento humano $(\mathrm{TH})$, transcendiendo los procesos operativos propios de la administración de personal, a los procesos emocionales y de desarrollo, donde se comienza a pensar en las personas como "ser humano", y que por ende llevan una vida personal, familiar y social que tiene impacto en el desempeño laboral (Restrepo y Arias, 2015). 
La competencia, la globalización, las nuevas tecnologías, el control de costos y los continuos cambios en los mercados, han sido la principal razón para la transformación de la gestión de TH, y son precisamente los desafíos competitivos. La gestión del TH constituye un factor clave para el desarrollo de la estrategia y consecución de metas y objetivos empresariales, así como la creación y el sostenimiento de ventajas competitivas (Beer, 1997; Calderón et al., 2010).

La calidad de vida en el trabajo (CVL) abarca todas aquellas condiciones relacionadas con el trabajo, como son los horarios, el sueldo, el ambiente laboral, los beneficios y servicios, las posibilidades de carrera y relaciones humanas, que pueden ser relevantes para la satisfacción y motivación laboral. Se trata pues de un concepto multidimensional usado para dar cuenta de la forma en que se produce la experiencia en sus condiciones objetivas como la seguridad, higiene, salario, etc., y las condiciones subjetivas como la forma en cómo lo vive (González et al., 2002).

Este concepto multidimensional se integra cuando el colaborador, a través del empleo y bajo su propia percepción, ve cubiertas las siguientes necesidades personales: soporte institucional, seguridad e integración al puesto de trabajo y satisfacción por el mismo, identificando el bienestar conseguido a través de su actividad laboral y el desarrollo personal alcanzado, así como la administración de su tiempo libre (González-Baltazar et al., 2010). Lo anterior se puede analizar mediante un análisis estructural, metodología estadística que utiliza un enfoque exploratorio y confirmatorio del análisis multivalente aplicado a una teoría estructural relacionada con un fenómeno determinado (Byrne, 1998).

Por lo anterior se hace necesario el estudio de elementos de gestión humana, como la calidad de vida laboral, en los sistemas de producción de leche, que permita esclarecer un punto de partida para mejorar la toma de decisiones y el desempeño productivo. El objetivo del trabajo fue explorar la calidad de vida en el trabajo y los factores asociados, de ordeñadores en lecherías especializadas del norte de Antioquia.

\section{MATERIAL Y MÉTODOS}

Se encuestaron 66 colaboradores que realizan labores de ordeño en sistemas de producción de leche en los municipios de Bello, Belmira, Don Matías, Entrerríos y San Pedro de los Milagros en el departamento de Antioquia, Colombia. La encuesta fue adaptada de la propuesta de González-Baltazar et al. (2010), la cual mide la calidad de vida en el trabajo en siete dimensiones: soporte institucional para el trabajo, seguridad en el trabajo, integración al puesto de trabajo, satisfacción por el trabajo, bienestar logrado a través del trabajo, desarrollo personal y administración del tiempo libre. La encuesta estuvo conformada por 44 preguntas. Las respuestas se presentaron en escala tipo Likert entre 1 (valor bajo) y 5 (valor alto).

Se llevó a cabo una validación de contenido la cual fue realizada por medio de la revisión temática del instrumento mediante las opiniones de cuatro expertos del área social y agropecuaria asegurando que las dimensiones medidas sean representativas del universo de las varia- bles de interés (Hernández et al., 2006) y la aplicación del cuestionario en tres ordeñadores, con el objetivo de identificar problemas en la comprensión del lenguaje o redacción de las preguntas, para garantizar sencillez y claridad de interpretación.

Para el análisis estadístico, se evaluaron las relaciones entre las variables por medio de un modelo de ecuaciones estructurales mediante análisis factorial exploratorio usando la librería psych (Revelle, 2017) del R-project (R Core Team, 2016), considerando dentro de cada factor las variables con un alpha de Cronbach superior a 0.70 y que la contribución de cada variable en cada factor generado sea superior a 0.53 según la carga de Schmid Leiman (Revelle, 2017). Posteriormente se realizó un análisis factorial confirmatorio incluyendo la variable exógena "deseo de permanecer" y las variables endógenas y latentes (o factores) generados en el análisis factorial exploratorio, validado con un índice ajuste comparativo (CFI) mayor que 0.90 , y la raíz cuadrada del error cuadrático medio aproximado del residuo (RMSEA) inferior al 0.04, mediante la librería "lavaan" (Rosseel, 2012), del software R-project (R Core Team, 2016).

Adicionalmente se analizó el grado de asociación de variables mediante correlaciones de Spearman superiores a 0.60 , y la variable "deseo de permanecer" fue asociada a todas las variables de la encuesta mediante un análisis de partición recursiva y árboles de regresión con la librería rpart (Therneau et al., 2015) del R-project software ( $R$ Core Team, 2016).

\section{RESULTADOS Y DISCUSIÓN}

Como se indica en la Tabla I, el análisis socio demográfico mostró que la mayoría de los ordeñadores encuestados tenían estudios de básica primaria (58\%), ratificando lo expuesto por Leibovich y Estrada (2008), quienes aseguraron que alrededor del $65 \%$ de la población en edad de trabajar, tenía únicamente primaria completa. Asimismo, la mayoría contaba con un contrato a término indefinido (77\%)y seguridad social (94\%), lo que indica el carácter formal de los empleos en la muestra evaluada; esto contrasta con lo expresado por Gómez et al. (2015) quienes afirmaron que aproximadamente un $50 \%$ de las personas que trabajan en Colombia lo hacen en la economía informal. Los autores expresaron además que los asalariados (empleos formales) tienen contratos de trabajo que les garantiza el acceso a los beneficios que se otorgan por ley. La mayoría de trabajadores se encontró en un rango de edad entre 41 y 50 años (30\%) y entre 31 y 40 años (27\%) y en menor frecuencia los mayores de 51 años (8\%).

Las variables observadas en las 7 dimensiones para abordar la CVL que tuvieron calificación menor de 4.0 fueron el "riesgo percibido", la "capacitación continua" y la "frecuencia con que se realizan los examenes de salud" que pertenecen a la dimensión "seguridad en el trabajo" y la variable "reconocimiento financiero" de la dimensión "soporte institucional", lo que denota oportunidades de gestión sobre estas, como mejoras, cambios, implementación de acciones preventivas/ correctivas, entre otras. 
Tabla I. Caracterización de sistemas especializados en producción de leche en Antioquia, Colombia, 2016 (Characterization of systems specialized in milk production in Antioquia, Colombia, 2016).

\begin{tabular}{|c|c|c|c|}
\hline & Concepto & Número & Frecuencia \\
\hline \multirow{2}{*}{$\begin{array}{l}\text { Certificación en } \\
\text { Buenas prácticas } \\
\text { ganaderas }\end{array}$} & No & 34 & 0.52 \\
\hline & Si & 32 & 0.48 \\
\hline \multirow{3}{*}{$\begin{array}{l}\text { Cobertura en } \\
\text { seguridad social }\end{array}$} & No & 4 & 0.06 \\
\hline & Si & 62 & 0.94 \\
\hline & Sin estudios & 10 & 0.15 \\
\hline \multirow{4}{*}{$\begin{array}{l}\text { Nivel de escola- } \\
\text { ridad }\end{array}$} & Primaria & 38 & 0.58 \\
\hline & Secundaria & 17 & 0,26 \\
\hline & Entre 16 y 23 & 11 & 0.17 \\
\hline & Entre 24 y 30 & 12 & 0.18 \\
\hline \multirow{3}{*}{$\begin{array}{l}\text { Edad de ordeña- } \\
\text { dores }\end{array}$} & Entre 31 y 40 & 18 & 0.27 \\
\hline & Entre 41 y 50 & 20 & 0.3 \\
\hline & Entre 51 y 60 & 5 & 0.08 \\
\hline \multirow{2}{*}{ Tipo de contrato } & Término indefinido & 51 & 0.77 \\
\hline & Término fijo & 8 & 0.12 \\
\hline \multirow{4}{*}{ Antigüedad } & Menos de 1 año & 24 & 0.36 \\
\hline & Entre 1 y 3 años & 22 & 0.33 \\
\hline & Entre 3 y 5 años & 13 & 0.2 \\
\hline & Más de 5 años & 4 & 0.06 \\
\hline
\end{tabular}

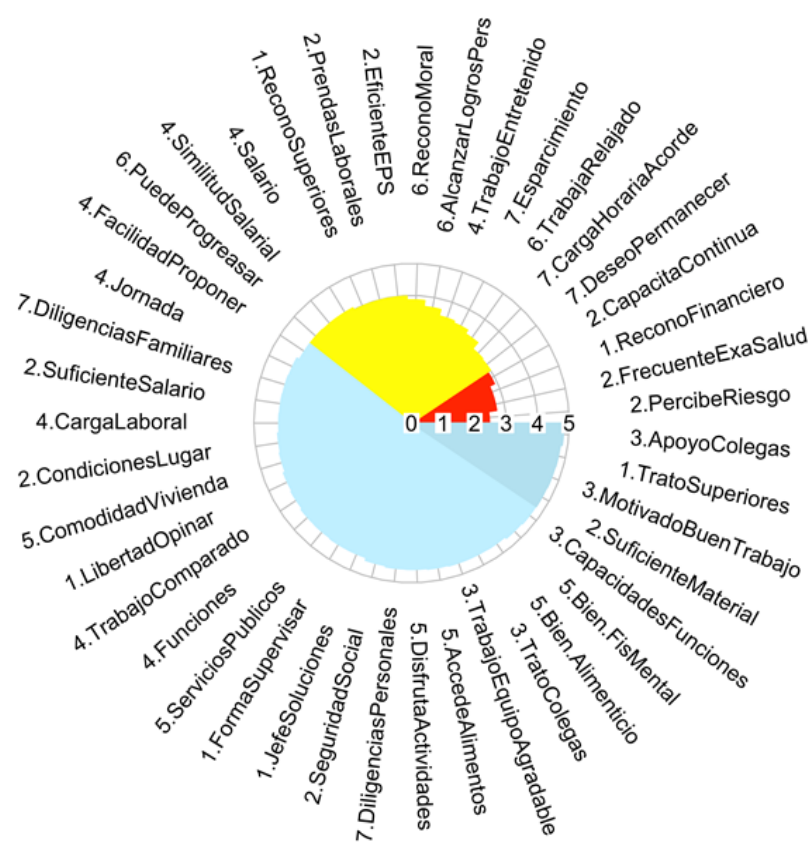

Figura 1. Apreciación de la calidad de vida laboral en las dimensiones: Soporte institucional (1), seguridad en el trabajo (2), integración al puesto de trabajo (3), satisfacción por el trabajo (4), bienestar logrado a través del trabajo (5), desarrollo personal (6) y administración del tiempo libre (7), en ordeñadores del norte de Antioquia (Appreciation of the quality of working life in the dimensions: Institutional support (1), safety at work (2), integration at the workplace (3), job satisfaction (4), wellness achieved through work(5), Personal development (6) and administration of leisure time (7), in milkers north of Antioquia.).
La seguridad en el trabajo se define como las características del empleo que se relacionan con las condiciones que brindan al colaborador firmeza en su relación con la institución (González-Baltazar et al., 2010).

Los resultados encontrados contradicen la apuesta realizada por el sector lechero colombiano, el cual se proyecta hacia la competitividad por medio de la gestión de calidad y la certificación en Buenas Prácticas Ganaderas (BPG), donde se requiere que el propietario o arrendatario del predio garantice que el personal se realice al menos un examen médico al año; además de capacitación en temas como higiene, seguridad y riesgo ocupacional, manejo de alimentos para animales, manejo animal, bioseguridad y uso seguro de medicamentos veterinarios y plaguicidas (Instituto Colombiano Agropecuario, 2007). Este comportamiento puede estar explicado por la racionalidad del productor al establecer que solo es benéfico incluir prácticas de gestión humana si estas se traducen en mejoras en la productividad o la rentabilidad de la actividad lechera; aspecto corroborado por Stup et al. (2006), al encontrar que no existe relación entre la rentabilidad de la producción de leche y las prácticas de recursos humanos de carácter técnico, como lo es la capacitación; haciéndose necesario contemplar variables más estrechamente relacionadas con la gestión humana tales como la satisfacción laboral de los empleados o el compromiso organizacional.

En la figura 1 se observa que se encontró un alto nivel de calidad de vida laboral en los ordeñadores encuestados del norte de Antioquia, debido a que en la mayoría de las dimensiones se encuentran variables positivamente valoradas. Vale la pena resaltar que las variables relacionadas con el individuo y la relación con su grupo laboral (compañeros y jefes) obtuvieron resultados positivos, mostrando satisfacción de los ordeñadores por las instrucciones recibidas para realizar el trabajo, la solidaridad y el apoyo, el trabajo en equipo, el poder expresar opiniones, la supervisión y el trato recibido por parte de colegas y superiores, entre otras; esto es importante ya que según Casas et al. (2002), la ausencia de contacto con otros colaboradores o la falta de apoyo y cooperación entre compañeros puede causar altos niveles de estrés, desmotivación y absentismo; asimismo la relación con el superior inmediato (estilo de supervisión, tipo de trato, comunicación, entre otras) pueden ser fuente principal de satisfacción o de tensión y estrés.

Estos hallazgos son relevantes ya que es contrario a lo que se esperaría, si se tiene en cuenta que, el sector lechero colombiano es un sector inmaduro en términos organizacionales, altamente informal, con limitaciones para el desarrollo de capacidades competitivas, caracterizado por un manejo empírico en el campo tecnológico y de la gestión empresarial; aspectos que pueden ser explicados porque son productores de escasos recursos económicos, cuyo objetivo principal es la supervivencia (Barrios et al., 2016); por lo tanto, en el afán de resolver el día a día, no se crea una conciencia real sobre aspectos de importancia como el bienestar y la retención de los colaboradores (Garcés et al., 2016). Para Ruiz et al. (2017), en las fincas con alto nivel de intensificación se implementan prácticas ambientales 
y sociales encaminadas a la sustentabilidad, buscando mayor productividad, lo que conlleva a mejor calidad de vida de los propietarios de los predios y sus colaboradores.

Se presentaron correlaciones mayores que 0.60 en los siguientes pares de variables: "trabajo compartido" con "funciones" (las dos pertenecen a la dimensión "satisfacción con el trabajo"), entre "funciones" con "bienestar alimenticio" (de la dimención bienestar) y bienestar alimenticio con "motivado con el buen trabajo"(dimensión integración al lugar de trabajo), entre "similitud salarial" con "salario" (las dos de la dimensión "satisfacción con el trabajo"), el "salario" con la "suficiencia salarial" (dimensión seguridad), la "suficiencia salarial" con la "posibilidad de progresar" (dimensión "desarrollo personal") y entre las variables "pueden progresar" con "acceso a los alimentos" (dimensión "bienestar").

Se puede destacar la relación entre la variable "bienestar alimenticio" (la cantidad y calidad de los alimentos para el ordeñador y su familia respecto a las expectativas de gusto y nutrición) y la "motivación para el trabajo". Esto puede explicarse desde la teoría de la motivación humana (Maslow, 1943), la cual define la motivación como la búsqueda de la satisfacción de la necesidad, que disminuye la tensión ocasionada por la misma; dicha teoría ubica la alimentación en la base

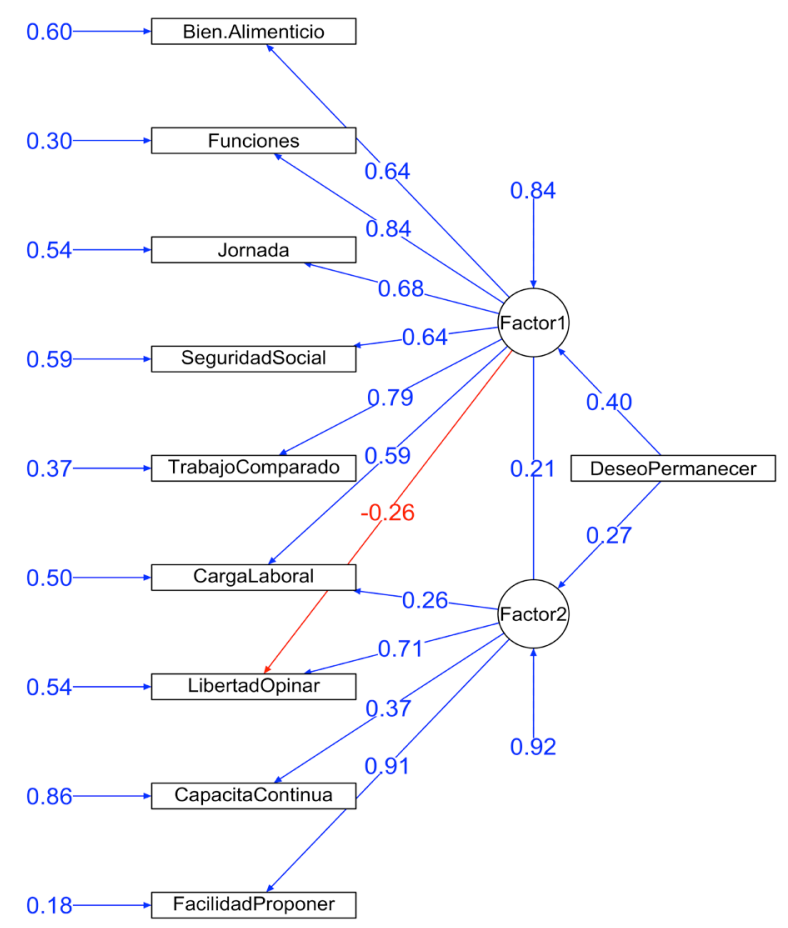

Figura 2. Análisis de ecuaciones estructurales de la calidad de vida de ordeñadores del Norte de Antioquia mediante la metodología de path-analysys (Analysis of structural equations of quality of life in the work of milkers in the Northern Antioquia, using path-analysys methodology). de la pirámide de necesidades, donde se encuentran las fisiológicas imprescindibles para la supervivencia, seguida por las de seguridad donde el individuo busca la estabilidad y protección para él y su familia, lo que conlleva a la otra relación ("salario" con el "progreso" y su "acceso a los alimentos"). Una vez estas están cubiertas, el ser humano aspira a alcanzar otras necesidades de mayor complejidad en el ámbito individual y colectivo.

Con la estructura de las siete dimensiones planteadas inicialmente se realizó un análisis factorial confirmatorio, donde se encontró que algunas variables presentaron un alfa de Cronbah inferior a 0.70 y un CFI inferior a 0.90 , por consiguiente se procedió a realizar un análisis factorial exploratorio con dos factores y posteriormente un análisis factorial confirmatorio. En la figura 2 se muestra la representación gráfica de las ecuaciones estructurales planteadas, en el análisis factorial confirmatorio con un índice de fiabilidad comparativo adecuado (CFI de 0.99) y una raíz del error cuadrático medio del residuo de aproximación adecuado (RMSEA de 0.03). Se puede observar que la intención de permanencia en el trabajo es una variable observada exógena dentro del modelo, pues no está siendo alterada por ninguna de las variables en el dendograma. Esta fue explicada por la presencia de dos constructos o variables latentes (dentro de los círculos) y por las covarianzas existentes entre ellas. Los colores verdes y la intensidad de estos en las líneas que unen las variables latentes, implican que la relación entre estas es positiva y que actúan de forma similar. El primer constructo "Factor 1" es el reflejo de seis variables observadas: bienestar alimenticio, carga laboral, funciones, jornada laboral, seguridad social y trabajo comparado con otras fincas. Todas impactan al constructo con una carga factorial superior a 0.60 ; destacando la variable satisfacción con las funciones, lo cual coincide con lo expresado por Flores y Madero (2012) quienes aseguraron que la satisfacción por el trabajo, es decir, la motivación intrínseca derivada de la tarea, influye en la intención de los colaboradores para permanecer en la organización, constituyéndose en un factor de la calidad de vida en el trabajo predictor de la intención de permanencia.

El segundo constructo "Factor 2" fue impactado por las variables observadas: capacitación continua, facilidad de proponer mejoras y libertad de opinar. En este caso, la variable observada que más aporta a la creación del constructo, es "facilidad de proponer", concordando con lo expuesto por Barragán et al. (2009), donde afirmaron que la mejor forma de retener a los empleados es ampliando sus responsabilidades y haciéndolos partícipes del funcionamiento de la organización, dándoles un espacio en la toma de decisiones, con el doble fin de que obtengan experiencia; lógicamente, este proceso es delicado y requiere de controles muy específicos.

Mediante partición recursiva fue posible determinar que las variables de mayor influencia en la intención de cambio de trabajo en los ordeñadores encuestados, son en su orden la percepción de que el trabajo puede poner en riesgo la salud y la disposición del jefe 
para conocer y resolver los problemas que se presentan en el trabajo.

La intención de permanecer en el trabajo será baja, si el ordeñador percibe un riesgo laboral por encima de 3.5 (figura 3). Como una forma de disminuir dicha percepción de afección y riesgos, la Resolución 2646 del Ministerio de la Protección Social (2008), establece disposiciones y define responsabilidades para la identificación, evaluación, prevención, intervención y monitoreo permanente de la exposición a factores de riesgo psicosocial en el trabajo y para la determinación del origen de las patologías causadas por el estrés ocupacional. El artículo $6^{\circ}$ de dicha resolución establece que los empleadores deberían evaluar los factores psicosociales del trabajo comprendiendo la identificación tanto de los factores de riesgo como de los factores protectores, con el fin de establecer acciones de promoción de la salud y prevención de la enfermedad en la población trabajadora.

A pesar del que ministerio establece que los empleadores deben identificar dichos factores existentes en la organización, entre estos los aspectos propios de la administración del recurso humano, como el estilo de liderazgo, las modalidades de pago y de contratación, el acceso a capacitaciones, mecanismos de evaluación y desempeño, y estrategias para manejar los cambios que afecten a las personas, entre otros (Ministerio de la Protección Social, 2008); este estudio evidencia que en el sector lechero existen elementos aún no trabajados en este ámbito, creando una brecha entre el deber ser y el hacer.

La variable que marcó la intención de quedarse en el trabajo actual fue la similitud salarial frente a otras

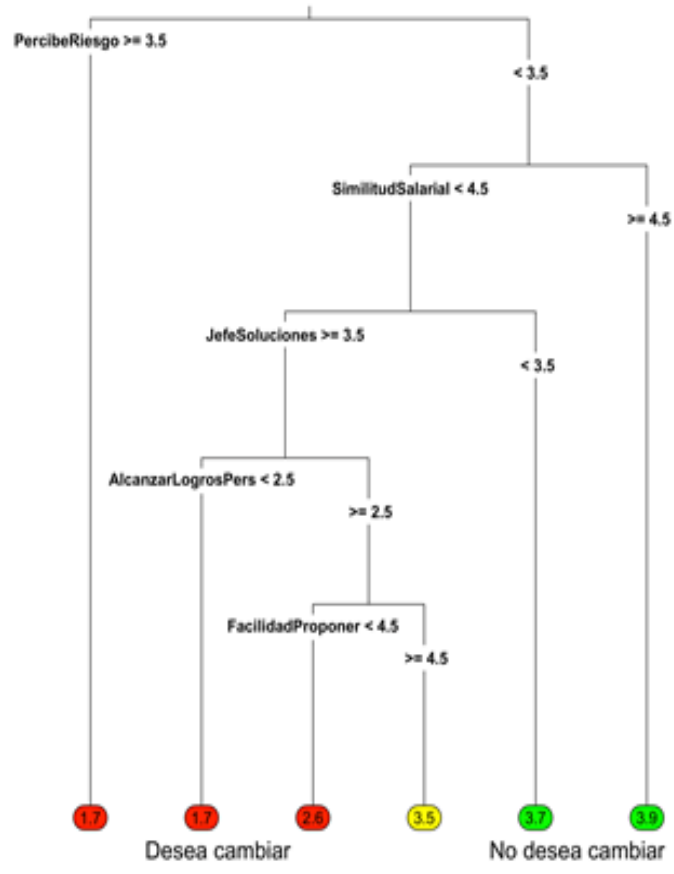

Figura 3. Intención de permanencia en el trabajo de ordeñadores en el norte de Antioquia (Intention to remain in the work of milkers in the north of Antioquia). fincas de la zona. Este comportamiento puede relacionarse con la Teoría de la Equidad (Adams, 1963), la cual postula que las personas distinguen entre las contribuciones que aportan en su trabajo y las compensaciones que reciben por estas, así como las que reciben otras personas; estableciéndose, por parte de los colaboradores, el criterio de equidad o la falta de este, y como consecuencia, se genera un estado de tensión que impulsa al colaborador intentar reducir este equilibrio. Este elemento cobra interés para los productores de leche, si se considera que es más desventajoso estar "muy por debajo del mercado" de lo que es ventajoso estar "muy por encima" de este. Estar notablemente por debajo del mercado hará que algunos solicitantes, a menudo los más deseables, rechacen la oferta; mientras que si se ajustan los salarios al precio de mercado, la elección se hará, generalmente, de forma multidimensional; donde factores distintos al salario pueden convertirse en ventajas competitivas (Rynes et al., 2004).

Cuando los colaboradores perciben que la relación entre su salario y su rendimiento no es justa, se puede producir insatisfacción, absentismo, deseos de abandonar la organización, bajo rendimiento o falta de dedicación, entre otras (Granados, 2011); por lo cual según los resultados encontrados, si la percepción sobre su salario es positiva (mayor a 4,5), al compararlo respecto a la remuneración que pagan otras fincas por hacer lo mismo, el ordeñador tendría la intención de permanecer en la organización. Sin embargo, estos resultados contradicen lo reportado por Pérez y Velásquez (2016), quienes encontraron que el tiempo dedicado para el descaso y el esparcimiento personal y con el grupo familiar son las causas principales de deserción o abandono del puesto de trabajo en sistemas de producción de leche, estando incluso por encima del salario.En los últimos tiempos, adicional al salario, la satisfacción sobre el ambiente de trabajo se ha configurado como un mecanismo de motivación; donde factores como el reconocimiento, la responsabilidad y la oportunidad de aprendizaje para mejorar el desempeño cobran gran importancia para muchos empleados (Srivastava y Barmola, 2011). Sin embargo, la remuneración salarial puede ser una motivación emocional que le permite al empleado incluirse en una organización que piensa en ellas como un ser que vive y necesita sostenerse bajo una calidad de vida digna (Garcés et al., 2016). Aunque el ofrecer al empleado salarios por encima de la media salarial, no es tan importante como optimizar los beneficios sociales, que se centran en la conciliación de los intereses particulares con los de la organización, la flexibilidad de turnos laborales, la calidad de vida, el ambiente laboral o que la organización lleve a cabo los valores que predica.

\section{CONCLUSIONES}

En este estudio exploratorio sobre la calidad de vida laboral del personal encargado del ordeño en sistemas de producción de leche del norte de Antioquia se evidencia que la percepción es positiva, destacando dimensiones como la integración al puesto de trabajo, el bienestar y desarrollo personal; los resultados contrastan con lo que se esperaría encontrar para el sector lechero, el cual es un sector inmaduro en temas 
organizacionales, altamente informal y con baja gestión empresarial.

La calidad de vida en el trabajo está afectada negativamente por los riesgos laborales, por la baja capacitación y por la falta de control médico, por lo tanto, estas variables se configuran como oportunidades en la gestión humana de los sistemas de producción de leche.

La intención de cambiar de trabajo es influenciada por la percepción de que el trabajo puede poner en riesgo la salud y la disposición del jefe para conocer y resolver los problemas que se presentan en el trabajo. La intención de permanencia en el trabajo actual está marcada por la variable de similitud salarial frente a otras fincas de la zona.

\section{AGRADECIMIENTOS}

Este artículo hace parte del proyecto "Fortalecimiento de la producción de la cadena láctea del distrito Norte Antioqueño", convenio N ${ }^{\circ}$ 2012AS180031 firmado entre la Secretaria de Agricultura y Desarrollo Rural del Departamento de Antioquia, La Universidad Nacional de Colombia (sede Medellín) y la Universidad de Antioquia, con recursos del Sistema General de Regalías- SGR. Se recibió el apoyo del comité para el desarrollo de la investigación- CODI (estrategia para la sostenibilidad ES84160119 grupo GaMMA 2016)

\section{BIBLIOGRAFÍA}

Adams, J.S. 1963. Towards an understanding of inequity. Journal of Abnormal Psychology, 67: 422-36.

Barragán, J.; Castillo, J.; Villalpando, P. y Guerra P 2009. Estrategias de retención de empleados eficientes: Importancia estratégica de la fidelización de los empleados en organizaciones internacionales. Innovaciones de Negocios, 6(1):33 - 43,

Barrios, D.; Restrepo-Escobar, F.J. y Cerón-Muñoz, M.F. 2016. Antecedentes sobre gestión tecnológica como estrategia de competitividad en el sector lechero colombiano. Livestock Research for Rural Development, 28:7.

Beer, M. 1997. The transformation of human resource function: resolving the tension between a traditional administrative and a new strategic role, Human Resource Management, 36(1):49-56.

Byrne, B.M. 1998. Structural Equation Modeling with LISREL, PRELIS, and SIMPLIS: basic concepts, applications, and programming. Mahwah: Lawrence Erlbaum Associates, Inc. N.J.

Calderón, G.; Naranjo, J.C. y Álvarez, C.M. 2010. Gestión humana en la empresa colombiana: sus características, retos y aportes. Una aproximación a un sistema integral Revista Cuadernos Latinoamericanos de Administración, 23(41):13-36.

Casas, J.; Repullo, J.R.: Lorenzo, S. y Cañas, J.J. 2002. Dimensiones y medición de la calidad de vida laboral en profesionales sanitarios. Revista de Administración Sanitaria, 5(23):143-60

Flores, R. y Madero, S.M. 2012. Factores de la calidad de vida en el trabajo como predictoras de la intención de permanencia. Acta universitaria, 22(2):24-31

Garcés, C.; Londoño, I.; Méndez, K. y Martínez, J. 2016. Retención de empleados, una estrategia para el éxito de las organizaciones. Revista Fundación Universitaria Luis Amigó, 3(1):108-15.

Gómez, M.S.; Galvis-Aponte, L.A. y Royuela, V. 2015. Calidad de vida laboral en Colombia: un índice multidimensional confuso. Documentos de trabajo sobre economía regional. Banco de la República. Centro de estudios económicos regionales-Cartagena, N. 230.
González-Baltazar, R.; Hidalgo-Santacruz, G.; Salazar, J.G. y PreciadoSerrano, M.L. 2010. Elaboración y validez del instrumento para medir calidad de vida en el trabajo "CVT-GOHISALO". C\&T, 12(36):332-40.

González, P.; Bravo, M.J. y Peiró, J.M. 2002. Calidad de vida laboral, En Peiró, J.M y Prieto F (eds.), Tratado de Psicología del Trabajo. Vol. II: La actividad laboral en su contexto, pp. 161-187.

Granados, I. 2011. Calidad de vida laboral: Historia, dimensiones y beneficios, Revista de Investigación en Psicología, 14(2):271-276.

Hernández, R.; Fernández-Collado, C. y Baptista, P. 2006. Metodología de la investigación. 4ta ed, Mc Graw Hill, México

Instituto Colombiano Agropecuario. 2007, Las buenas prácticas ganaderas en la producción de leche, en el marco del Decreto 616, hitp://www.ica.gov.co/getdoc/7eceb128-553c-45d8-955db3a660e80257/Publicacion-23.aspx

Leibovich, J. y Estrada, L. 2008. Competitividad del sector agropecuario colombiano. EN: Informe final de competitividad 2008-2009. Consejo Privado de Competitividad. https://issuu.com/cnpcolombia/docs/ informe_nacional_competitividad

Maslow, A.H. 1943. A theory of human motivation. Psychological Review, 50(13):370-96.

Ministerio de Protección Social. 2008. Resolución 2646 por la cual se establecen disposiciones y se definen responsabilidades para la identificación, evaluación, prevención, intervención y monitoreo permanente de la exposición a factores de riesgo psicosocial en el trabajo y para la determinación del origen de las patologías causadas por el estrés ocupacional. Bogotá, Colombia, 17 de julio de 2008

Pérez, C.A. y Velásquez, O.H. 2016. Calidad de Vida en el TrabajoGOHISALO- en hatos lecheros en pastoreo en Don Matías y La Unión, Colombia. Journal of agriculture and animal sciences 5(2):32-48.

R Core Team. 2016. A language and environment for statistical computing. Vienna, Austria. Disponible en: http://www.r-project.org/.

Restrepo, F. y Arias, F. 2015. Las prácticas de gestión del talento humano en empresas agropecuarias del sector bananero en Colombia, Journal of Agriculture and Animal Science, 4(2):20-32

Revelle, W. 2017. Psych: procedures for personality and psychological research, Northwestern University, Evanston, Illinois, USA.

Rosseel, Y. 2012. Lavaan: An \{R\} Package for Structural Equation Modeling, J. Stat. Softw, 48(2): 1-36 http://www.jstatsoft.org/v48/i02/.

Ruiz, J.F.; Cerón-Muñoz, M.F.; Barahona-Rosales, R. y Bolivar-Vergara D.M. 2017. Caracterización de sistemas de producción bovina de leche según el nivel de intensificación y su relación con variables ambientales y sociales asociadas a la sustentabilidad, Livestock Res earch and Rural Development, 29: 1

Rynes, S.L.;Gerhart, B. and Minette, K.A. 2004. The importance of pay employee motivation: discrepancies between what people say and what they do. HRM, 43(4):381-394 Srivastava, S.K. y Barmola, K.C. 2011 . Role of motivation in higher productivity. Mgmt. Insight Journal, VII (1):88-99

Stup, R.E.; Hyde, J. and Holden, L.A. 2006. Relationship between selected human resource management practices and dairy farm Performance. Journal of Dairy Science, 89:1116-1120

Therneau, T.; Atkinson, B. and Ripley, B. 2015. Rpart: Recursive Partitioning and Regression Trees, R package version 4.1-10. https:// CRAN.R-project.org/package=rpart. 